\title{
A Review of the Applications of Biochemicals Interference in Medical Imaging with the Synthesis of Contrast Nanoparticles and Marker Design (PROBE)
}

\author{
Zahra Nouri $1, * \mathbb{D}$ \\ 1 School of Medicine, Tehran University of medical sciences, Tehran, Iran \\ * Correspondence: zahranouritums@gmail.com (Z.N.);
}

Scopus Author ID 57190046716

Received: 21.03.2021; Revised: 15.05.2021; Accepted: 20.05.2021; Published: 18.06.2021

\begin{abstract}
One of the main focuses in diagnostic medicine is molecular imaging. Nanoparticles allow us to image different components of a high-contrast molecule efficiently. In this study, various factors that should be considered when synthesizing contrast nanoparticles are discussed. Some of the most important examples are highlighted. This research has been done theoretically and in terms of content analysis method (Content Analysis) by searching for keywords nanotechnology, molecular imaging, drug delivery methods, gene therapy, and contrast nanoparticles in google, PubMed, Science direct, scholar, and Scopus websites. After the search, ten articles were selected from the obtained articles, and this article was written based on them. Most of the report's nanoparticles produce new contrast agents, especially molecular imaging and cellular processes detection. Have been taken. The advantages of using these nanoparticles are the ability to produce high contrast, ease of integration of multiple properties, long circulation time in the blood, and the ability to carry high-volume materials (such as drugs). The basics and nanoparticle production methods have grown and expanded over the years, so more complex examples of nanoscale contrast agents such as paramagnetic particles, macrophages with quantum dots, quantum dots, machines that can make matter Atomic and molecular parts have been reported. MRI of microemulsions has also been used to examine blood vessels and deliver medication. The use of contrast nanoparticles provides more details about the processes of a disease and its effects. The fabrication of these materials has improved dramatically over the past decade by creating various functions in them. However, there are many areas for biocompatibility, efficacy, specificity, and diagnosis of further diseases. In general, contrast nanoparticles can be synthesized for a specific function, with specific properties to perform a specific program.
\end{abstract}

Keywords: Nanotechnology; Molecular Imaging; Magnetic Resonance Imaging (MRI); Drug delivery methods; Gene therapy.

(C) 2021 by the authors. This article is an open-access article distributed under the Creative Commons Attribution (CC BY) license (https://creativecommons.org/licenses/by/4.0/).

\section{Introduction}

The introduction deals with this issue. Molecular imaging can be one of the main areas of focus in diagnostic medicine, early detection imaging of diseases, early detection of molecular disease. As reference [1] provided, providing vital information about process pathology will help us.

Imaging at the cellular, molecular level relies heavily on developing complex contrast agents required for the cell's biological detection of processes [2]. Contrast nanoparticles have a significant advantage over molecular-based contrast agents, including creating very high contrast (such as quantum dots), integrating different properties such as different contrast types, 
longer blood circulation, and the ability to charge more material. Allow us to photograph different components of a high-contrast molecule efficiently [1,2]. As a result, exciting results from reported molecular imaging include detecting specific cell types, factors that multiple imaging techniques can detect, and factors contributing to the advancement of imaging of new cellular systems. There are other studies on this subject; for example: In cardiovascular patients, nanoparticles have been used for molecular imaging [3].

The effective synthesis of contrast nanoparticles for molecular imaging requires close attention to the test sample's required properties. Synthesis of nanoparticles with contrast application, treatment includes surface coating optimization, marking, appropriate size, and a high degree of improved biocompatibility [3, 4]. In this study, the current state of the synthesis of optimized contrast nanoparticles has been investigated, and some examples of factors that have been effective in their highly advanced synthesis have been pointed out [4-9].

\section{Materials and Methods}

This research is of theoretical type and method by content analysis (Content Analysis) by searching for keywords nanotechnology, molecular imaging, drug delivery methods, gene therapy, and contrast nanoparticles in science, google scholar, PubMed, direct and Scopus is done. After searching, ten articles were selected from the obtained articles, and this article was written based on them.

\section{Results and Discussion}

The latest technologies in the design of contrast nanoparticles. The latest molecular imaging methods with contrast nanoparticles to produce suitable contrast using fluorescent, radioactive, paramagnetic, super-magnetic, or dense electron damping materials. Nanoparticles have been used for properties such as therapeutic [2]. Contrast enhancing or therapeutic compounds can be placed in the core of the particle, inside the coating, or attached to the nanoparticle's surface. This flexibility in incorporating various types of contrast materials and Combining contrast and therapeutic materials in nanoparticles is very helpful in selecting clinical diagnosis cases. Detection of nanoparticles by imaging methods such as MRI and fluorescence allows them to be detected in the target tissue or cell types. A combination of contrast agents and therapeutic agents is known as prostatic and allows us to image drug, protein, or gene transfer. Several nanoparticles can have many properties and factors, such as contrast agents. Therapeutic agents, fluorescence, and heat He easily placed the targets in them. A research [3] report on multifunctional nanoparticles for imaging macrophages in atherosclerosis, including micelles with quantum dots enclosed in the nucleus. The micelles are coated around the quantum dots with a composite film, polyethylene. Phospholipidmodified glycol, Gadolinium-labeled lipid (for contrast MRI), and polyethylene phospholipid glycol have an active functional group. By controlling lipid / amphiphilic input, gadolinium's ratio to fluorophore can be evenly controlled to mark. These properties are in the particle's composition in two steps, which can be done only after the purification step. This method is simpler and more efficient than synthesizing several steps of a molecule, including the target group, contrast agent, and fluorophore. A team from the University of Washington $[4,5]$ extensively studied carbon fluorescence-based microemulsions as contrast agents targeting atherosclerotic plaque components such as integrin- $3 \alpha v \beta$, fibrin, or collagen III. The carbon fluorescence nucleus allows "Bright spots" in MRI imaging with 
Use the drug inclusion system as hydrophobic in the core. In this example, the antiangiogenic drug fumagillin, composed of gadolinium with a phospholipid coating, was tested for contrast in MRI. Target particles use a specific peptide bond with $\beta$ - $\alpha \alpha \beta$ integrin, which increases the contrast in the study of blood vessels. In the therapeutic approach, the authors twice injected atherosclerosis into the arterial wall of the model rabbit. MRI images obtained four hours after the first injection showed a significant volume of contrast material in the aorta, while after the second injection (one week later), the increase in contrast intensity in the aorta was low. See Figure 1.

This effect is the lack of angiogenesis of fumagillin in the primary injection. Therefore the reduction of the density of small vessels and, as a result, the lack accumulation of the agent has occurred after applying secondary microemulsion [6].
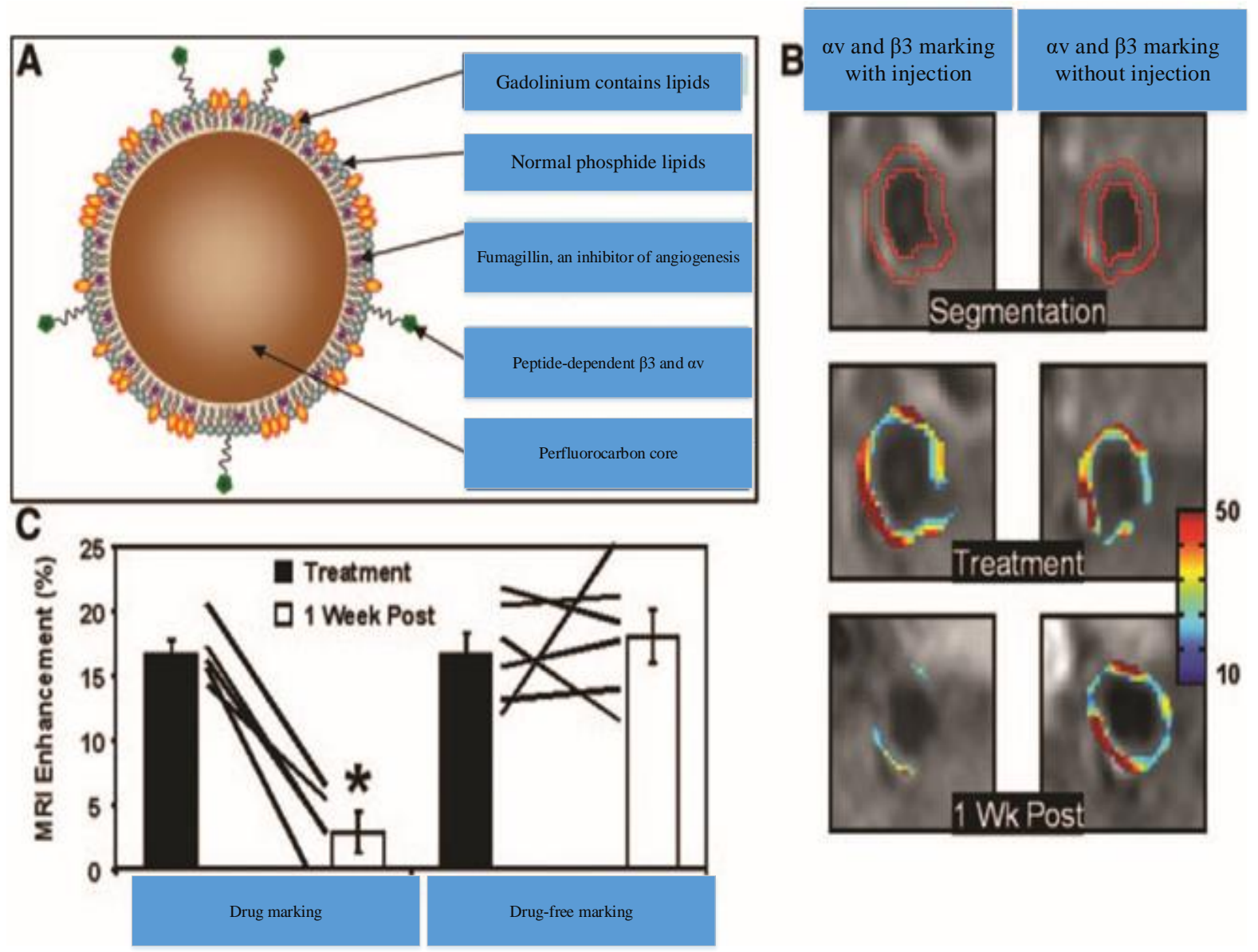

Figure 1. The volume of contrast material in the aorta after the first and second injections.

Iron oxide nanoparticles are sensitive contrast agents for MRI. New iron oxide particles have been used for gene expression and gene therapy. Cross-sectional nanoparticles with dextran amine shells were first added to Cy5/5, a near-infrared drug. Transfer to the polymeristoylated membrane of arginine peptide (MPAP), and finally, siRNA was used to turn off the gene.

The synthesis of new nanoparticles allows them to be detected by both MRI and fluorescence methods and is efficiently absorbed by the cell and capable of silencing certain genes. In initial experiments, siRNA binding to the green fluorescence protein was used to see Becomes. Mice were infected with two tumors, one expressing GFP and the other red fluorescent protein (RFP), and MRI and fluorescence examined the uptake of particles in these tumors in the laboratory and the body. Fluorescence imaging showed that GFP granite was reduced by up to $85 \%$ by siRNA iron oxide particles, while fluorescent protein expression 
remained unchanged in red [6]. Besides, therapeutic versions of nanoparticles have been developed in which siRNA attached to survivin, an inhibitor of the apoptotic protein, has been observed. In this case, the adsorption of nanoparticles was assessed using MRI and fluorescence imaging 24 hours after injection. After four injections and after two weeks, the survivin level in these mice's tumors decreased to $3 \%$ of the sample group level. It has arrived. Besides, apoptosis and necrosis, which was increased in the tumor, were reduced in treated mice [6]. These can be found through immunofluorescence and histology, so siRNA iron oxides are effective in treating cancer (see Figure 2).
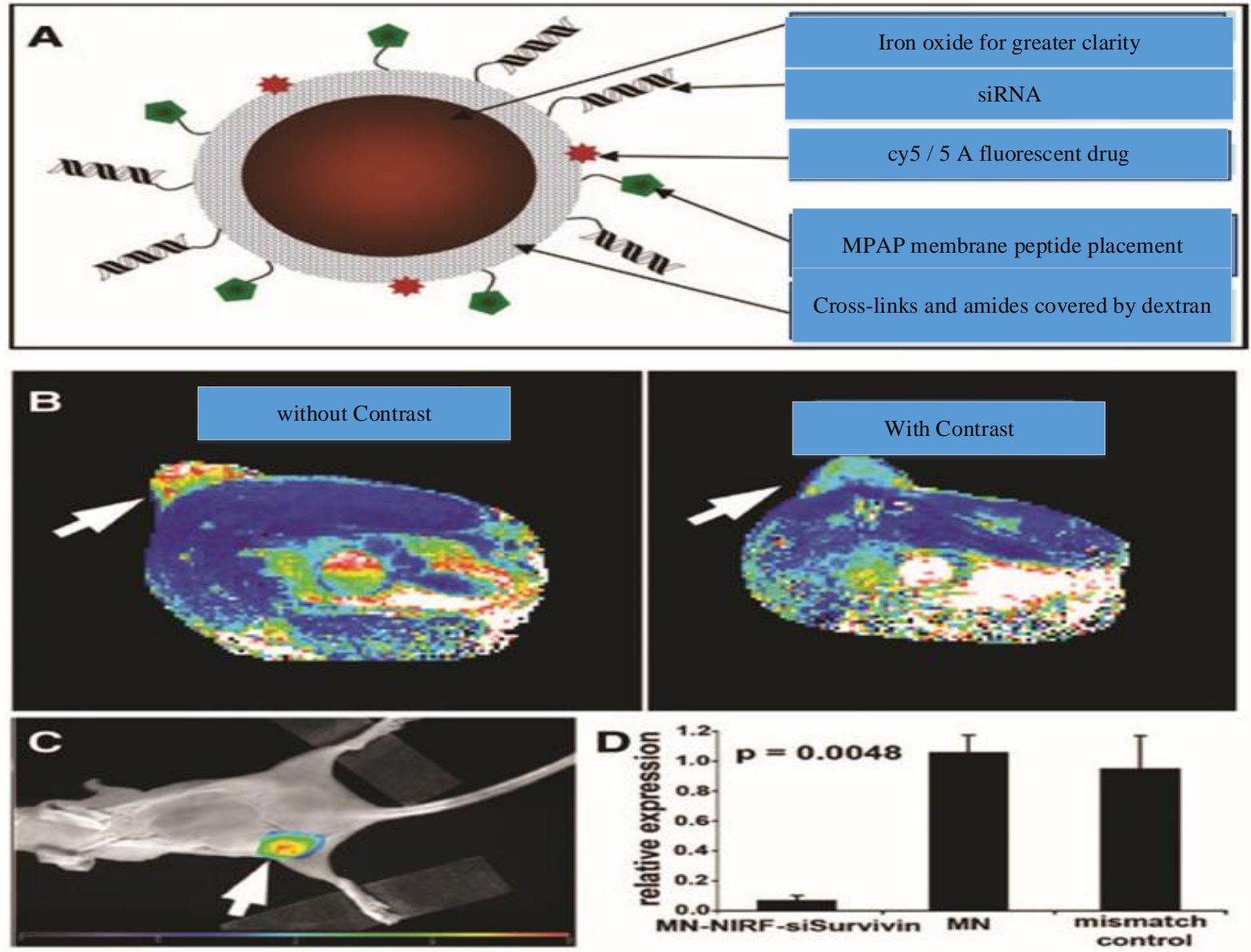

Figure 2. SiRNA iron oxides in the treatment of cancer.

Selection of nanoparticles: a wide range of nanoparticles used as contrast agents) [710] For different imaging methods, the need for nanoparticles with different properties to produce contrast is appropriate. This section briefly describes the nanoparticles used for each method. Liposomes are adhesive gadolinium ions, microemulsion micelles, lipoproteins, viruses, and carbon nanotubes suitable for contrast-weighted MRI-T1 using the paramagnetic properties of these elements.

Superparamagnetic iron oxides are widely used to create contrast by MRI weight (* T2) [1]. Iron or gadolinium can be used as the contrast agent, which varies depending on the application. Although gadolinium nanoparticles' detection sensitivity is generally lower than that of iron oxide nanoparticles, gadolinium creates positive contrast. It easily accumulates in specific tissues [11]. Iron oxides have negative contrast and poor signal, and because there are many cases of signal loss in MRI images, it reduces our confidence in materials with iron oxide accumulations. Creating an image with a new sequence to produce a positive contrast of iron oxide may alleviate this problem. Several factors in the image's weight have led to the use of 
iron oxide or gadolinium nanoparticles being investigated. For example, iron oxide sensitivity is very useful if the target is photographed at very low concentrations [12]. Iron oxides are also suitable for tissue imaging, producing homogeneous MRI signals with predictable structures, including the brain [13]. In areas of the body where structures are less predictable and signal vacuum is common (including the abdomen), the advantage of gadolinium-based agents has been used as a generator of positive contrast. [13] Quantum dots are excellent contrast materials. They are fluorescent for imaging; they have a wide excitation range and a narrow diffusion interval, they are highly efficient, are transparent, and do not fade [14]. Light in the near-infrared range (650 to $900 \mathrm{~nm}$ ) and in which the tissue absorption range is low is defensible. For fluorescence tomography, light must pass through the thin tissue thickness, but it does not matter if the microscope Confocal, for example, is effective only to absorb a drop that is about ten $\mu \mathrm{m}$ thick [15].

In contrast, dense electron-based nanoparticles with high atomic numbers such as iodine, bismuth, or gold have been proposed for CT scans, and most researchers have examined their material in solid nanoparticles $[15,16]$. Still, some have provided liposomes that Inside, a solution containing iodinated molecules is trapped. Gold nanoparticles have become a popular choice for CT scans, although it is unclear whether these materials are suitable for use. Research [17], based on solid nanoparticles iodine, has been reported to be specific to rabbit atherosclerosis by macrophages, suggesting the possibility of showing highly sensitive molecular imaging in vivo using CT scan [18]. CT scan is thought to be instrumental. Ultrasound requires contrast agents to create a secondary spectrum of sound waves to the marker head, typically using micron-sized water-insoluble gas bubbles such as perfluorobutane to stabilize the bubble. We need proper coverage, but coverage reduces flexibility and builds up. Better contrast is much needed. The result is that the coatings currently in use must have a combination of stability and flexibility. Multilayer vesicles are also used as contrast agents in ultrasound, although their echogenic response depends on the gas trapped in them. Microemulsions have also been used in sonography [19]. Conceptually, drugs and contrast agents can attach to nanoparticle surfaces, either embedded in the wall or loaded into the nucleus.

Types of coatings: Most of the materials used to create contrast in molecular imaging are low biocompatibility, short half-life in the circulatory system, rapid excretion, reduced stability, and high toxicity. Therefore, many attempts have been made to provide suitable compounds [20]. Also, materials such as phospholipids, dextran, polyvinyl pyridine, polyethylene glycol, or silica have been used as coatings. At present, new nanoparticles are labeled. These coatings increase the water absorption of nanoparticles, reducing their aggregation, improving their physical properties and beneficial effects. Otherwise, the uncoated nanoparticles are known as foreign bodies. Therefore, by covering and deceiving the body's defense mechanism and avoiding phagocytosis, their half-lives significantly increase in the blood. Regulation is widely used as a coating strategy in nanoparticles. It has been used to increase their half-life in blood circulation by preventing the rapid removal of nanoparticles by the reticuloendothelial system. High durability in the circulatory system helps nanoparticles remain in the body longer and reach and connect to the target organ. Silica is a delivery platform for nanoparticles for all types. Consignments such as drugs, genes, proteins, or contrast agents are used. Research [21, 22] show how silica particles containing quantum dot can be pegylated with a lipid coating to improve their biocompatibility compared to silica particles, unmodified surfaces, lipid-coated particles, and lipid paramagnetism. The nanoparticles have shown less 
toxicity with their lipid coating, a tenfold increase in circulating half-life, and no lumps in the lungs [22].

In summary, the lipid coating improves biocompatibility. However, it still needs to be further explored how silica is metabolized and excreted from the body. An alternative strategy to synthetic coatings using natural nanoparticles such as viruses or lipoproteins has been explored to prevent recognition by the body's defense system. For this purpose, two methods have been proposed: in the lipid ion/surface phase, natural particles can be modified and surrounded by contrast ions and drugs $[9,23]$. The second method is a new combination of organic and unnatural materials, including inorganic nanoparticles. The nucleus is a virus or lipoprotein. The second method by research [23] is Iron oxide, quantum dots, or gold nanoparticles were incorporated into high-density lipoprotein and shown by negative staining and electron microscopy. Iron oxide, quantum dots, and high-density lipoprotein nanoparticles are called QD-HDL, FeO-HDL, and AU-HDL. Paramagnetic phospholipids or fluorescent phospholipids combined with particles so that each MRI and fluorescence technique and CT scan by AU-HDL have created a contrast. Atherosclerosis in mice is shown by MRI, fluorescence imaging, and computed tomography (see Figure 3).

Marking strategy: There are several methods for marking nanoparticles. There are significant blood vessels in the tissues and cancerous tissues and inflammatory diseases such as atherosclerosis. Vessels with large and dense nodes increase nanoparticles' accumulation due to the long half-life of blood due to the magnetic resonance's permeability and stability of ma In cases where the examination of damaged tissue vessels without any target ligand is required, nanoparticle coverage and increased circulatory system stability should be ensured. Another inactive method is to mark the use of dextran as nanoparticle coating [8, 24]. Macrophages target Dextran-coated iron oxide nanoparticles. Various molecules, including antibodies, antibody fragments, proteins, peptides, peptide bonds, aptamers, sugars, and small molecules, can be labeled. Active is attached to the nanoparticle. Marking the above with a ligand is important; besides these, other factors must also be considered; specific antibodies are expensive. After Binding to particles up to $10 \mathrm{~nm}$ in diameter, the antibody to certain species may trigger an immune response. Smaller antibodies, although cheaper, may not be used for labeling. As a result, short peptide sequence strands are used to identify a specific target [25]. Methods developed by Weislider et al. [26], a large collection of target molecule nanoparticles that have been labeled with cheap and simple ligands against various cells and, after identifying the appropriate ligand, have considered its association with nanoparticles. The biotin-streptavidin linkage has often been considered but has evoked an immune response in the patient, so ligands have been attached to nanoparticles using various methods, and covalent bonding prevents an immune response [27].

Effects of size: The size of nanoparticles plays an essential role in various cases, including the types of cells targeted, material load, oscillation, material repellency, and quality in contrast. The size of nanoparticles has a significant impact on the biological distribution of those particles and thus show The pathology of inflamed tissues of the body with highly permeable vessels such as tumors or atherosclerotic plaques can be examined by the penetration of nanoparticles, which is an acceptable result if the nanoparticles of the face are small. The size range of nanoparticles is determined by their synthesis method. Considerable efforts have been made to develop specific particle production by various methods, resulting in mineral nanocrystals' size and shapes, such as iron oxide, gold nanoparticles, silica, or quantum dots. Lipid-based nanoparticles can be precisely controlled. However, the particle diameter with the 
same nuclei will vary depending on the coating type $[28,29]$. The size of the nanoparticles is very important in their excretion from the body. Research [30] has shown the decomposition and clearance of nanoparticles in the kidney. The fluorescence excretion of nanoparticles from the kidneys is shown to be equal to or less than $5.5 \mathrm{~nm}$, while particles larger than that are mainly shown by the reticuloendothelial system and finally where the nanoparticles are trapped in the liver and spleen and where They are metabolized and excreted.

On the one hand, if nanoparticles accumulate in the body, toxicity occurs. On the other hand, if the nanoparticles are small enough, they are excreted by the renal system. Their halflife is reduced [30]. Changes in the size of nanoparticles change their contrast. For example, in the propagation of fluorescent quantum dots, the nanoparticles' wavelength also increases with increasing wavelength.

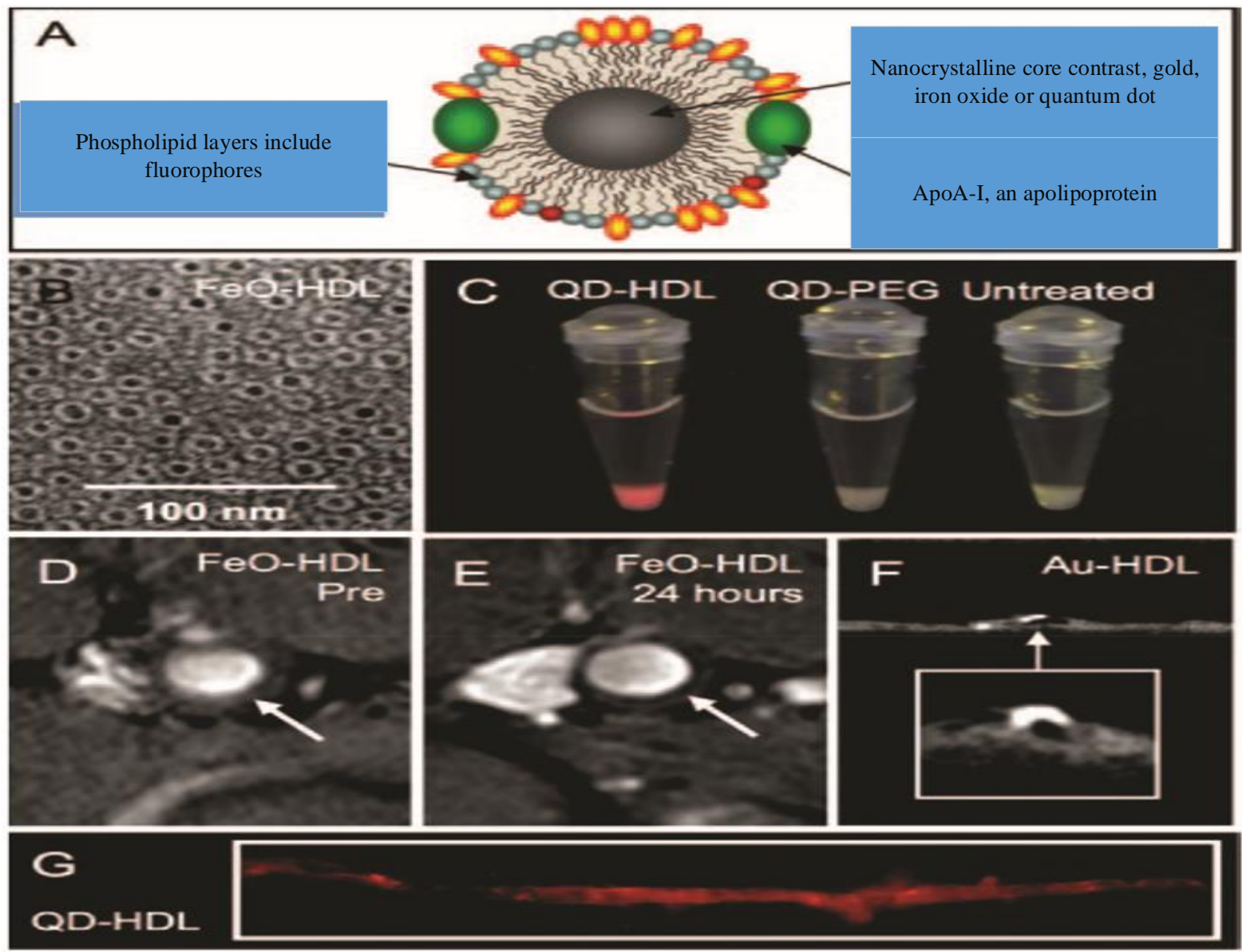

Figure 3. Particles in vitro in MRI atherosclerosis

Therefore, the desired and appropriate wavelength can be achieved by adjusting the appropriate sizes for the propagation of these particles. Iron oxide particles in the range between one $\mathrm{nm}$ and one $\mu \mathrm{m}$ have excellent contrast in magnetic imaging particles, but these particles are helpful to us in the emerging technique of magnetic imaging particles when they are larger than $20 \mathrm{~nm}$. The size of nanoparticles affects the biological distribution, material loading capacity, and contrast. Therefore, how to determine particle size is an essential question that scientists face. Many different methods are currently available, but choosing a suitable method is essential because each technique gives us specific information. Electron microscopy, for example, provides the diameter of inorganic nuclei but often does not specify the size and diameter of the coating. However, the hydrodynamic diameter varies depending on the sample concentration and buffer type [23]. 
Effect of nanoparticle concentration: Scientifically, there is a relationship between the size of nanoparticles and their surface density, that the smaller the size of nanoparticles, the higher their surface density. According to research on nanoparticle concentrations, it has been concluded that surface nanoparticle concentrations directly affect the probability of the target cell's chance so that by increasing the surface concentration, the probability of detecting and targeting target cells increases. This, in turn, reduces time and increases accuracy [23-25]. Other effects of concentration include the attachment of target cells to the target material, which can be a drug or imaging material that a specific receptor of the target cell can be placed on the target material most inclined with the target cell and causes a targeted and strong connection. The higher the concentration of material on the surface, the stronger the connection with the target cell, and its sensitivity increases [24, 26-28].

It is best to coat the nanoparticles with biocompatible materials to obtain the Food and Drug Administration's approval when synthesizing nanoparticles. For this purpose, a watersoluble polymer is naturally used for coating [28, 29]. Nanoparticles such as liposomes or micelles do not need to be coated, as they are highly biocompatible. The structural synthesis of solid nanoparticles makes this possible. The nanoparticles synthesized in the organic phase are usually insoluble in water and require a second coating to produce biological properties[3034]. Fortunately, there are several efficient ways to do this. This is typically done using dual or molecular polymers such as phospholipids [22, 30]. Another vital issue before nanoparticle synthesis is how to add properties such as target groups or fluorophores to the nanoparticle surface. The dextran-coated iron and crosslinked with the modified amine group can be coupled to target groups, fluorophores, and metal ions [34-39]. To label using this approach, a small amount of phospholipid reactivity was included. In this particular example, maleimide was used, including amines, carboxylic acids, pyridyl dithiopropionite, and biotin, are also available.

The nanoparticles used to increase and improve the contrast should not be structurally toxic [22]. Finally, there may be more restrictions on their use, and larger particles should not remain in the liver and spleen. It also determines how the body reacts to nanoparticles and how they break down. Can these nanoparticles be used in humans? The US Food and Drug Administration will ultimately determine whether nanoparticles can be used in humans. It is a fact that there are still no standard guidelines for the use of nanoparticles, which has complicated the roadmap for the future. However, many efforts have been made to produce bioparticles that are compatible with the body. For example, research [29] has done extensive laboratory assays to explain nanoparticles' effect on various cellular processes.

On the other hand, some nanoparticles that decompose into non-toxic substances in this way can be considered safe. If iron oxide particles are used as free ions (injected) because of Restriction of the body occurs in the decomposition of iron toxicity. However, suppose iron oxide enters the body in a crystalline state. In that case, it decomposes slowly and under control into finer particles of iron. As another option, completely inert materials such as gold can be used [1]. If nanoparticles after decomposition into components. Toxic (such as quantum dots) converted to cadmium fragments (care should be taken when using them for clinical use. However, these nanoparticles are very common in clinical applications).

\section{Conclusions}

By synthesizing nanoparticles while considering the conditions and properties of use, they can be used as a basis for the production of high-impact contrast materials. The fabrication 
of these materials has improved dramatically over the past decade by creating various functions in them, such as more efficient labeling, biocompatibility, and the achievement of appropriate imaging enhancements. In general, contrast nanoparticles can be synthesized for a specific function at this stage of development, with specific properties to perform a specific program. However, there are many areas for biocompatibility, effectiveness, specificity, and diagnosis of more diseases. The standardization of the structure of clinical/paraclinical research needs further development. Finally, nanotechnology will continue producing and exploiting new nanoparticles in medical imaging with better properties and have become a will to develop medical imaging that requires new nanoparticle formulations as contrast agents.

\section{Funding}

This research received no external funding.

\section{Acknowledgments}

The author, at this moment, thanks the Tehran University of medical sciences for their kind support.

\section{Conflicts of Interest}

The authors declare no conflict of interest.

\section{References}

1. Zhao, Y.; He, Z.; Zhang, Q.; Wang, J.; Jia, W.; Jin, L.; Zhao, L.; Lu, Y. 880 nm NIR-Triggered Organic Small Molecular-Based Nanoparticles for Photothermal Therapy of Tumor. Nanomaterials 2021, 11, 773, https://doi.org/10.3390/nano11030773.

2. Prabhakar, N.; Belevich, I.; Peurla, M.; Heiligenstein, X.; Chang, H.-C.; Sahlgren, C.; Jokitalo, E.; Rosenholm, JM. Cell Volume (3D) Correlative Microscopy Facilitated by Intracellular Fluorescent Nanodiamonds as Multi-Modal Probes. Nanomaterials 2021, 11, 14, https://doi.org/10.3390/nano11010014.

3. Knights, O.; Freear, S.; McLaughlan, J.R. Improving Plasmonic Photothermal Therapy of Lung Cancer Cells with Anti-EGFR Targeted Gold Nanorods. Nanomaterials 2020, 10, 1307, https://doi.org/10.3390/nano10071307.

4. Marpu, S.B.; Kamras, B.L.; MirzaNasiri, N.; Elbjeirami, O.; Simmons, D.P.; Hu, Z.; Omary, M.A. SingleStep Photochemical Formation of Near-Infrared-Absorbing Gold Nanomosaic within PNIPAm Microgels: Candidates for Photothermal Drug Delivery. Nanomaterials 2020, 10, 1251, https://doi.org/10.3390/nano10071251.

5. Siddique, S.; Chow, J.C.L. Application of Nanomaterials in Biomedical Imaging and Cancer Therapy. Nanomaterials 2020, 10, 1700, https://doi.org/10.3390/nano10091700.

6. Yan, Y.; Ding, H. pH-Responsive Nanoparticles for Cancer Immunotherapy: A Brief Review. Nanomaterials 2020, 10, 1613. https://doi.org/10.3390/nano10081613.

7. Shimizu, K.; Iyoda, T.; Yamasaki, S.; Kadowaki, N.; Tojo, A.; Fujii, S.-i. NK and NKT Cell-Mediated Immune Surveillance against Hematological Malignancies. Cancers 2020, 12, 817, https://doi.org/10.3390/cancers12040817.

8. Pilla, L.; Maccalli, C. Immune Profiling of Cancer Patients Treated with Immunotherapy: Advances and Challenges. Biomedicines 2018, 6, 76, https://doi.org/10.3390/biomedicines6030076.

9. Zocchi, M.R.; Tosetti, F.; Benelli, R.; Poggi, A. Cancer Nanomedicine Special Issue Review Anticancer Drug Delivery with Nanoparticles: Extracellular Vesicles or Synthetic Nanobeads as Therapeutic Tools for Conventional Treatment or Immunotherapy. Cancers 2020, 12, 1886, https://doi.org/10.3390/cancers12071886. 
10. Korir, D.K.; Gwalani, B.; Joseph, A.; Kamras, B.; Arvapally, R.K.; Omary, MA; Marpu, S.B. Facile Photochemical Syntheses of Conjoined Nanotwin Gold-Silver Particles within a Biologically-Benign Chitosan Polymer. Nanomaterials 2019, 9, 596, https://doi.org/10.3390/nano9040596.

11. Tan, N.P.B.; Lee, C.H.; Li, P. Green Synthesis of Smart Metal/Polymer Nanocomposite Particles and Their Tuneable Catalytic Activities. Polymers 2016, 8, 105, https://doi.org/10.3390/polym8040105.

12. Li, H.-J.; Li, P.-Y.; Li, L.-Y.; Haleem, A.; He, W.-D. Gold Nanoparticles Grafted with PLL-b-PNIPAM: Interplay on Thermal/pH Dual-Response and Optical Properties. Molecules 2018, 23, 921, https://doi.org/10.3390/molecules23040921.

13. Caro, C.; Gámez, F.; Quaresma, P.; Páez-Muñoz, J.M.; Domínguez, A.; Pearson, J.R.; Pernía Leal, M.; Beltrán, A.M.; Fernandez-Afonso, Y.; De la Fuente, J.M.; Franco, R.; Pereira, E.; García-Martín, M.L. Fe3O4-Au Core-Shell Nanoparticles as a Multimodal Platform for in Vivo Imaging and Focused Photothermal Therapy. Pharmaceutics 2021, 13, 416, https://doi.org/10.3390/pharmaceutics13030416.

14. Kim, M.; Kim, G.; Kim, D.; Yoo, J.; Kim, D.-K.; Kim, H. Numerical Study on Effective Conditions for the Induction of Apoptotic Temperatures for Various Tumor Aspect Ratios Using a Single Continuous-Wave Laser in Photothermal Therapy Using Gold Nanorods. Cancers 2019, 11, 764, https://doi.org/10.3390/cancers11060764.

15. Leung, J.P.; Wu, S.; Chou, K.C.; Signorell, R. Investigation of Sub-100 nm Gold Nanoparticles for LaserInduced Thermotherapy of Cancer. Nanomaterials 2013, 3, 86-106, https://doi.org/10.3390/nano3010086.

16. Ahijado-Guzmán, R.; Sánchez-Arribas, N.; Martínez-Negro, M.; González-Rubio, G.; Santiago-Varela, M.; Pardo, M.; Piñeiro, A.; López-Montero, I.; Junquera, E.; Guerrero-Martínez, A. Intercellular Trafficking of Gold Nanostars in Uveal Melanoma Cells for Plasmonic Photothermal Therapy. Nanomaterials 2020, 10, 590, https://doi.org/10.3390/nano10030590.

17. Liu, B.; Cao, W.; Cheng, J.; Fan, S.; Pan, S.; Wang, L.; Niu, J.; Pan, Y.; Liu, Y.; Sun, X.; Ma, L.; Song, J.; $\mathrm{Ni}$, J.; Cui, D. Human natural killer cells for targeting delivery of gold nanostars andbimodal imaging directed photothermal/photodynamic therapy and immunotherapy. Cancer Biol Med. 2019, 16, 756-760, https://doi.org/10.20892/j.issn.2095-3941.2019.0112.

18. Prabhakar, N.; Peurla, M.; Shenderova, O.; Rosenholm, JM. Fluorescent and Electron-Dense Green Color Emitting Nanodiamonds for Single-Cell Correlative Microscopy. Molecules 2020, 25, 5897, https://doi.org/10.3390/molecules25245897.

19. Claveau, S.; Bertrand, J.-R.; Treussart, F. Fluorescent Nanodiamond Applications for Cellular Process Sensing and Cell Tracking. Micromachines 2018, 9, 247, https://doi.org/10.3390/mi9050247.

20. Sotoma, S.; Iimura, J.; Igarashi, R.; Hirosawa, K.M.; Ohnishi, H.; Mizukami, S.; Kikuchi, K.; Fujiwara, T.K.; Shirakawa, M.; Tochio, H. Selective Labeling of Proteins on Living Cell Membranes Using Fluorescent Nanodiamond Probes. Nanomaterials 2016, 6, 56, https://doi.org/10.3390/nano6040056.

21. Naya, M.; Sato, C. Pyrene Excimer-Based Fluorescent Labeling of Cysteines Brought into Close Proximity by Protein Dynamics: ASEM-Induced Thiol-Ene Click Reaction for High Spatial Resolution CLEM. Int. J. Mol. Sci. 2020, 21, 7550, https://doi.org/10.3390/ijms21207550.

22. Ramezani, F.; Razmgir, M.; Tanha, K.; Nasirinezhad, F.; Neshasteriz, A.; Bahrami-Ahmadi, A.; Hamblin, M.R.; Janzadeh, A. Photobiomodulation for spinal cord injury: A systematic review and meta-analysis. Physiology \& Behavior 2020, 3,112977, https://doi.org/10.1016/j.physbeh.2020.112977.

23. Tashakori-Miyanroudi, M.; Rakhshan, K.; Ramez M, Asgarian, S.; Janzadeh, A.; Azizi, Y.; Seifalian, A.; Ramezani, F. Conductive carbon nanofibers incorporated into collagen bio-scaffold assists myocardial injury repair. International Journal of Biological Macromolecules 2020, 163, 1136-46, https://doi.org/10.1016/j.ijbiomac.2020.06.259.

24. Zarepour, L.; Gharaylou, Z.; Hadjighassem, M.; Shafaghi, L.; Majedi, H.; Behzad, E.; Hosseindoost, S.; Ramezani, F.; Nasirinezhad, F. Preliminary study of analgesic effect of bumetanide on neuropathic pain in patients with spinal cord injury. Journal of Clinical Neuroscience 2020, 81, 477-84, https://doi.org/10.1016/j.jocn.2020.10.010.

25. Ramez, M.; Ramezani, F.; Nasirinezhad, F.; Rajabi, H. High-intensity interval training increases myocardial levels of Klotho and protects the heart against ischaemia-reperfusion injury. Experimental physiology 2020, 105(4), 652-65, https://doi.org/10.1113/EP087994.

26. Ghanimati, R.; Rajabi, H.; Ramezani, F.; Ramez, M.; Bapiran, M.; Nasirinezhad, F. The effect of preconditioning with high-intensity training on tissue levels of G-CSF, its receptor and C-kit after an acute myocardial infarction in male rats. BMC cardiovascular disorders 2020, 20(1), 1-9, https://doi.org/10.1186/s12872-020-01380-w. 
27. Vahed, M.; Ramezani, F.; Tafakori, V.; Mirbagheri, V.S.; Najafi, A.; Ahmadian, G. Molecular dynamics simulation and experimental study of the surface-display of SPA protein via Lpp-OmpA system for screening of IgG. AMB Express 2020, 10(1), 1-9, https://doi.org/10.1186/s13568-020-01097-1.

28. Behroozi, Z.; Ramezani, F.; Janzadeh, A.; Rahimi, B.; Nasirinezhad, F. Platelet-rich plasma in umbilical cord blood reduces neuropathic pain in spinal cord injury by altering the expression of ATP receptors. Physiology \& Behavior 2021, 228, 113186, https://doi.org/10.1016/j.physbeh.2020.113186.

29. Ramezani, F.; Neshasteh-Riz, A.; Ghadaksaz, A.; Fazeli, S.M.; Janzadeh, A.; Hamblin M.R. Mechanistic aspects of photobiomodulation therapy in the nervous system. Lasers in Medical Science 2021, 24, 1-8, https://doi.org/10.1007/s10103-021-03277-2.

30. Saffarpour, S.; Janzadeh, A.; Rahimi, B.; Ramezani, F.; Nasirinezhad, F. Chronic nanocurcumin treatment ameliorates pain-related behavior, improves spatial memory, and reduces hippocampal levels of IL-1 $\beta$ and $\mathrm{TNF} \alpha$ in the chronic constriction injury model of neuropathic pain. Psychopharmacology 2021, 238(3), 87786, https://doi.org/10.1007/s00213-020-05739-x.

31. Sharifi, M.; Nazarinia, D.; Ramezani, F.; Azizi, Y.; Naderi, N.; Aboutaleb, N. Necroptosis and RhoA/ROCK pathways: molecular targets of Nesfatin-1 in cardioprotection against myocardial ischemia/reperfusion injury in a rat model. Molecular Biology Reports 2021, 48(3), 2507-18, https://doi.org/10.1007/s11033-021-06289$\mathrm{x}$.

32. Hoseini, Z.S.; Hajizade, A.; Easton, A.J.; Ahmadian, G.; Ramezani, F. A meta-analysis of the efficiency of metal nanoparticles in vaccine delivery against infectious disease. Nanomedicine 2021, 16(6), 481-95, https://doi.org/10.2217/nnm-2020-0358.

33. Šaulienè, I.; Šukienè, L.; Daunys, G.; Valiulis, G.; Lankauskas, A.; Kokina, I.; Gerbreders, V.; Gavarāne, I. Detection and Microscopy of Alnus glutinosa Pollen Fluorescence Peculiarities. Forests 2019, 10, 959, https://doi.org/10.3390/f10110959.

34. Virant, D.; Turkowyd, B.; Balinovic, A.; Endesfelder, U. Combining Primed Photoconversion and UVPhotoactivation for Aberration-Free, Live-Cell Compliant Multi-Color Single-Molecule Localization Microscopy Imaging. Int. J. Mol. Sci. 2017, 18, 1524, https://doi.org/10.3390/ijms18071524.

35. Zhao, L.; Choi, J.; Lu, Y.; Kim, S.Y. NIR Photoregulated Theranostic System Based on Hexagonal-Phase Upconverting Nanoparticles for Tumor-Targeted Photodynamic Therapy and Fluorescence Imaging. Nanomaterials 2020, 10, 2332, https://doi.org/10.3390/nano10122332.

36. Manivasagan, P.; Bharathiraja, S.; Santha Moorthy, M.; Mondal, S.; Nguyen, T.P.; Kim, H.; Phan, T.T.V.; Lee, K.D.; Oh, J. Biocompatible Chitosan Oligosaccharide Modified Gold Nanorods as Highly Effective Photothermal Agents for Ablation of Breast Cancer Cells. Polymers 2018, 10, 232, https://doi.org/10.3390/polym10030232.

37. Luo, M.; Cheng, W.; Zeng, X.; Mei, L.; Liu, G.; Deng, W. Folic Acid-Functionalized Black Phosphorus Quantum Dots for Targeted Chemo-Photothermal Combination Cancer Therapy. Pharmaceutics 2019, 11, 242, https://doi.org/10.3390/pharmaceutics11050242.

38. Lim, K.; Kim, H.K.; Le, X.T.; Nguyen, N.T.; Lee, E.S.; Oh, K.T.; Choi, H.-G.; Youn, Y.S. Highly Red LightEmitting Erbium- and Lutetium-Doped Core-Shell Upconverting Nanoparticles Surface-Modified with PEGFolic Acid/TCPP for Suppressing Cervical Cancer HeLa Cells. Pharmaceutics 2020, 12, 1102, https://doi.org/10.3390/pharmaceutics12111102.

39. Wang, B.-Y.; Liao, M.-L.; Hong, G.-C.; Chang, W.-W.; Chu, C.-C. Near-Infrared-Triggered Photodynamic Therapy toward Breast Cancer Cells Using Dendrimer-Functionalized Upconversion Nanoparticles. Nanomaterials 2017, 7, 269, https://doi.org/10.3390/nano7090269. 